\title{
Human Intestinal Condition Identification Based-on Blended Spatial and Morphological Feature using Artificial Neural Network Classifier
}

\author{
Ummi Athiyah $^{\text {a, } 1, * \text {, Arif Wirawan Muhammad }}{ }^{\text {b, c, } 2, \text { Ahmad Azhari }}{ }^{\text {d,3 }}$ \\ ${ }^{a}$ Department of Data Science, Institut Teknologi Telkom Purwokerto \\ Jl DI Pandjaitan 128 Karangreja, Banyumas 53147, Indonesia \\ ${ }^{b}$ Department of Informatics, Institut Teknologi Telkom Purwokerto \\ Jl DI Pandjaitan 128 Karangreja, Banyumas 53147, Indonesia \\ ${ }^{c}$ Fakulti Sains Komputer dan Teknologi Maklumat, Universiti Tun Hussein Onn Malaysia \\ Jl Delta 6 Parit Raja, Johor, 86400, Malaysia \\ ${ }^{d}$ Department of Informatics, Universitas Ahmad Dahlan \\ Jl Ringroad Selatan, Tamanan, Banguntapan, Bantul, Yogyakarta 55166, Indonesia \\ ${ }^{1}$ ummi@ittelkom-pwt.ac.id *; ${ }^{2}$ arif@ittelkom-pwt.ac.id; ${ }^{3}$ ahmad.azhari@tif.uad.ac.id \\ * corresponding author
}

Article history:

Received 15 June 2020

Revised 23 June 2020

Accepted 30 June 2020

Published online 17 August 2020

Keywords:

intestinal condition

blended spatial

morphological feature

neural-networks

classifier
Colon cancer is a type of disease that attacks the intestinal walls cell of humans. Colorectal endoscopic screening technique is a common step carried out by the health expert/gynecologist to determine the condition of the human intestine. Manual interpretation requires quite a long time to reach a result. Along with the development of increasingly advanced digital computing techniques, then some of the weaknesses of the manually endoscopic image interpretation analysis model can be corrected by automating the detection process of the presence or absence of cancerous cells in the gut. Identification of human intestinal conditions using an artificial neural network method with the blended input feature produces a higher accuracy value compared to the artificial neural network with the non-blended input feature. The difference in classifier performance produced between the two is quite significant, that is equal to $0.065(6.5 \%)$ for accuracy; $0.074(7.4 \%)$ for recall; 0.05 $(5.0 \%)$ for precision; and $0.063(6.3 \%)$ for f-measure.

This is an open access article under the CC BY-SA license (https://creativecommons.org/licenses/by-sa/4.0/).

\section{Introduction}

Colorectal cancer is a type of cancer that attacks the intestinal walls cell of humans. Wisconsin Reporting System (WRS), states that the type of colorectal cancer is the third highest cause of death after types of lung cancer and breast cancer, with a case of death of $9.5 \%$ of the total world population [1]. In Indonesia, colorectal cancer itself is ranked as the third cause of death after breast cancer and cervical cancer. Therefore, the abnormalities in the human intestinal wall need to be identified early to minimize the growth of cancerous cells that are more virulent, which can cause death. Health screening, such as endoscopy, is a simple step to detect abnormal growths in human intestinal cells wall [2][3]. Also, the process of early detection plays an essential role in health practitioners/gynecologists to determine the prognosis and type of treatment that patients must receive [4]. The right prognosis is accompanied by the right dosage of the drug to help speed the recovery of patients from colorectal cancer outbreaks.

Endoscopic screening technique is a common step carried out by the health experts/gynecologist to determine the condition of the human intestine by inserting a camera through the rectum to get an intestine picture [5]. The result of endoscopic screening is a digital image of the area around the intestinal wall [6]. During this time, the interpretation of endoscopic images is carried out manually or taken by the naked eye, thus requires quite a long time to interpret and produce results. This 
manual interpretation is the cause of the long duration of time needed by the patient to find out the results of endoscopic screening [7]. This image interpretation has a weakness; it depends on the gynecologist's expertise and experience [8]. Along with the development of increasingly advanced digital computing techniques, then some of the weaknesses of the manually endoscopic image interpretation analysis model mentioned previously can be corrected by automating the detection process of the presence or absence of cancer cells in the gut, by utilizing digital image processing techniques supported by the machine method learning. Automating the detection process can speeding up in production results and minimizing errors arising from the manual analysis model of endoscopic image interpretation.

\section{Method}

The identification of the intestinal condition to find cancerous colon condition in this study was divided into several steps presented in Fig. 1. The stages of Fig. 1 are explained in the following subsection points:

The first step, retrieving dataset is to get a colorectal endoscopic dataset. The colorectal endoscopy dataset consists of 200 image files generated from the colorectal endoscopic screening process with a .png format with VGA resolution $(640 \times 480$ pixels $)$. The images in the dataset are divided into two categories: (1) endoscopic images for normal intestinal wall conditions and (2) endoscopic images for abnormal intestinal wall conditions that are the origin of cancerous conditions. Examples images of normal intestinal wall conditions can be seen in Fig. 2.

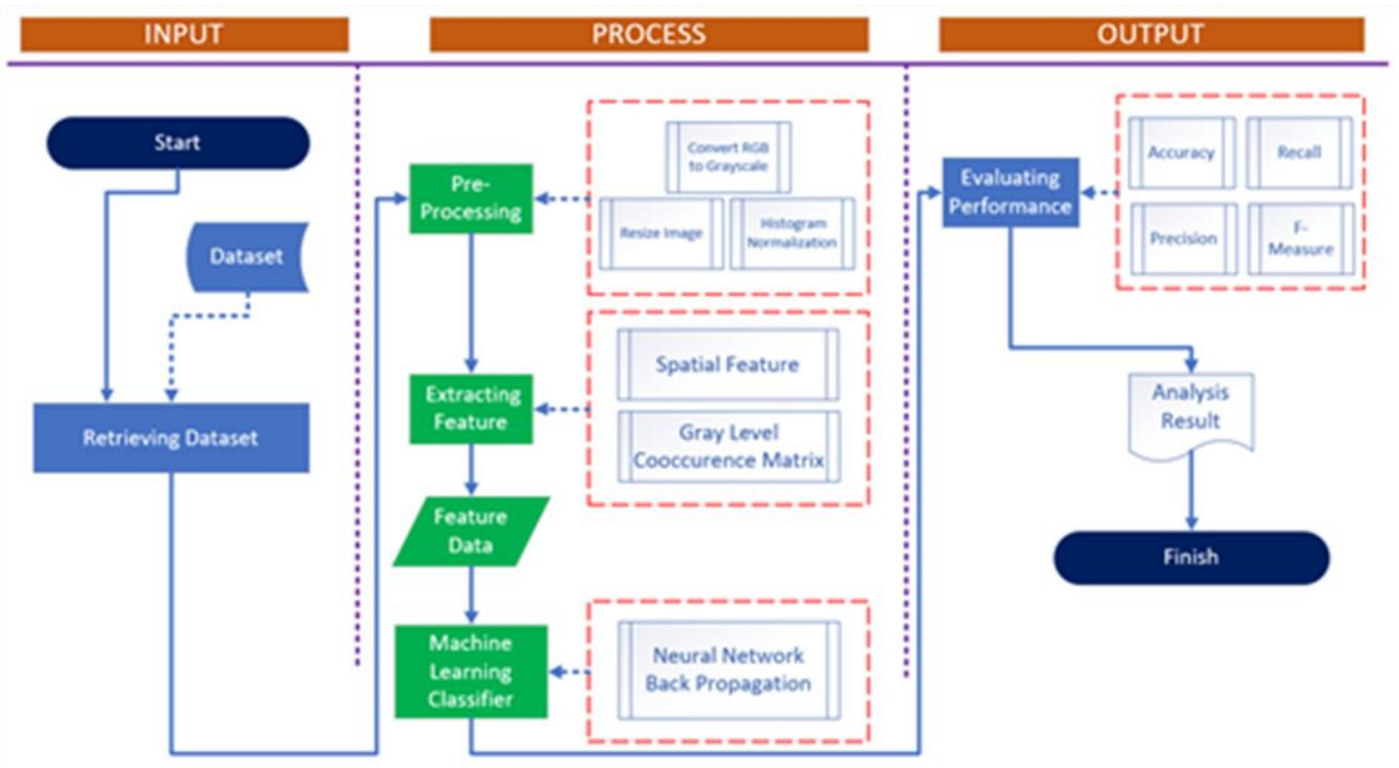

Fig. 1. Colon cancer identification approach

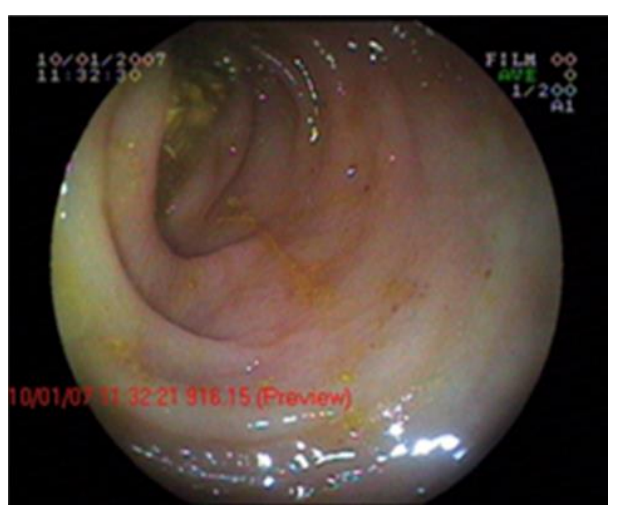

Fig. 2. Endoscopic imagery of normal intestinal wall conditions 


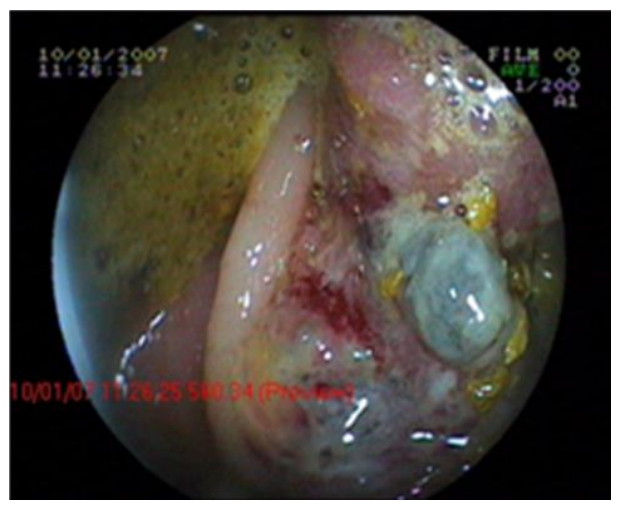

Fig. 3. Endoscopic imagery of abnormal intestinal wall conditions

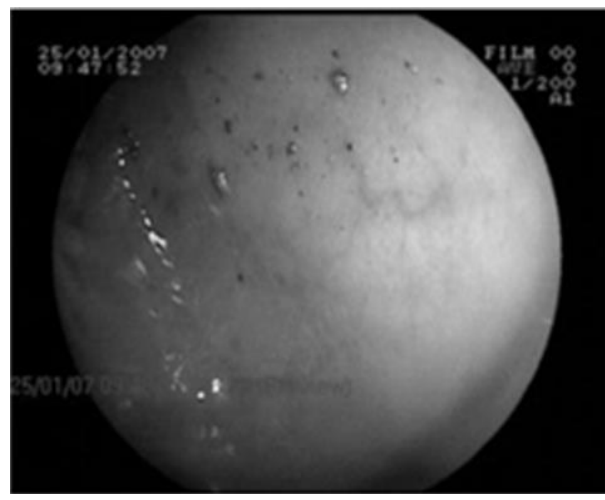

Fig. 4. Converted image from RGB to grayscale

While examples of endoscopic images for abnormal intestinal wall conditions can be seen in Fig. 3. The overall endoscopic dataset was obtained from the Internal Medicine Laboratory Dr. Sardjito Hospital, Yogyakarta-Indonesia, under supervision of Dr. Putut Bayupurnama Sp.PD.

The preprocessing aims out to ensure that the original image is ready for further processing at the feature extraction stage. Preprocessing also plays a vital role in avoiding bias in the output of a machine learning classifier. Preprocessing in this study is divided into several stages:

\section{Convert Image RGB to Grayscale}

At this stage, the conversion of RGB channel images into grayscale channels is carried out by using the formula presented in (1) by taking the red color conformity of $30 \%$, green by $59 \%$, and blue by $11 \%$ [2]. The RGB conversion extracts features of spatial images in the form of circularity, aspect ratio, triangularity, and cooccurence matrix values.

$$
\text { grayscale }=0.30 * r+0.59 * g+0.11 * b
$$

where $\mathrm{R}$ indicates the value of the red channel, $\mathrm{G}$ the green channel, and $\mathrm{B}$ the Blue channel, an example of the results of converting an RGB image of endoscopic images under normal conditions to grayscale is presented in Fig. 4.

\section{Image Resize}

The image size reduction speeds up image processing and reduces the computational burden by change the pixel size of the original image from $640 \times 480$ pixels to $320 \times 240$ with the .png format [9].

\section{Histogram Normalization}

At this stage, the image histogram normalization is carried out which aims to equalize the brightness and contrast patterns that are owned by the image and the distribution of pixel intensities [10]. Histogram normalization is carried out using the formula presented in (2). 
Equation (2) ensures that the interval of gray image values [0-255] is mapped in the range of values 0 until 1 only [11].

$$
i_{\text {new }}=\sum_{i=0}^{K=1} P(i)
$$

where $i_{\text {new }}$ is the value of pixel normalization mapping from a range [0-255] to range [0-1]; $n_{i}$ is the number of pixels in an image $I(x, y)$ with gray level degrees of $(i)$; while $p(i)$ represents the pixel probability in gray level degrees of $(i)$.

Feature extraction from endoscopic images is carried out to retrieve relevant information from each image so it can be used as input to the machine learning classifier. In this study, relevant information extracted from endoscopic imagery includes morphological information and spatial information. Morphological information, taken based on the size of circularity that is the result of the division between the pixel area value and the pixel perimeter on the region of interest (ROI). The circularity formula is presented in (3) [6].

$4 \pi * \frac{\text { area }}{\text { perimeter }}$

Whereas spatial feature information, extracted based on the co-occurrence matrix, which will produce information includes:

\section{Energy}

Energy is a measure of pixel conformity in an image. Energy reflects the degree of texture smoothness of an image. The lower the energy value, the rougher the surface texture of the image and vice versa [12]. The calculation of the energy value is presented in (4)

$$
\text { energy }=\sum_{i, j} P(i, j)^{2}
$$

\section{Contrast}

Contrast value is the simple comparison between foreground objects and image background. Contrast is a unit of local image variation values [12][13]. The calculation of the contrast value is presented in (5)

$$
\text { contrast }=\sum_{i, j}|i-j|^{2} P(i, j)^{2}
$$

\section{Correlation}

Correlation is a gray-level linearity value of two or more adjacent pixels in an image [14]. The calculation of the correlation value is presented in (6)

$$
\text { correlation }=\sum_{i, j} \frac{\left(i-\mu_{i}\right)\left(j-\mu_{j}\right) P_{i, j}}{\sigma_{i} \sigma_{j}}
$$

\section{Homogeneity}

It is a value of the distance between elements in the co-occurrence matrix in gray images [11]. The calculation of the homogeneity value is presented in (7)

$$
\text { homogeneity }=\sum_{i, j} \frac{P_{i, j}}{1+|i-j|}
$$

where $P(i, j)$ is the elements of the co-occurrence matrix; $\mu_{i}$ and $\mu_{j}$ express the mean value and $\sigma_{i}$ and $\sigma_{j}$ reflect the standard deviation in row $i$ and column $j$ in.

The machine learning model built in this study is an artificial neural network that utilizes the backpropagation function. The architecture of artificial neural networks is presented in Table 1. At the input layer of the artificial neural network architecture, 4 neurons and 5 neurons are used following the number of feature extractions from the colonic endoscopy image. While the hidden layer / intermediate layer uses 9 and 11 neurons following the equation stated by [12][15] that the use of a hidden layer of $2 n+1$ (where $n$ is the number of input neurons) can accelerate the training process and the generalization results of neural networks. At the output layer, the binary-shot coding concept is used where 1 represents the condition of the cancerous image, while 0 represents the normal image condition [16]. 
Table 1. The architecture of Artificial Neural Network

\begin{tabular}{llll}
\hline Point & Layer information & Number of neuron & Activation function \\
\hline A & Layer Input & 4 Neurons a and 5 Neurons ${ }^{\mathrm{b}}$ & - \\
$\mathrm{B}$ & $\begin{array}{l}\text { Layer Intermediate } \\
\text { (Hidden) }\end{array}$ & $\begin{array}{l}9 \text { Neurons a and 11 Neurons } \\
\text { b }\end{array}$ & Logsig \\
C & Layer Output & 2 Neurons (using binary-shot coding) & Purelin \\
\hline
\end{tabular}

${ }^{a}$ Non-blended feature (spatial feature only)

${ }^{\mathrm{b}}$ Blended feature (spatial \& morphological feature)

Validation of the classification results is Accuracy, Recall, Precision, and F-Measure. The three parameters are obtained from true positive (TP), true negative (TN), false positive (FP), and falsenegative (FN) metrics [17].

1. TP is a condition when the input " $A$ " identified by machine learning matches the ground truth " $A$ ".

2. TN is a condition when the "non- $A$ " input identified by machine learning matches the "non$A$ " groundtruth.

3. FP is a condition when input " $A$ " is identified by machine learning as a "non- $A$ " groundtruth.

4. FN is a condition when the input "non- $A$ " is identified by machine learning as the " $A$ " groundtruth.

From the indicators mentioned above, an equation can be formed, stating the accuracy presented in (8) until (11) [18].

$$
\begin{aligned}
& \text { accuracy }=\frac{(\mathrm{TP}+\mathrm{TN})}{(\mathrm{TP}+\mathrm{TN}+\mathrm{FP}+\mathrm{FN})} \\
& \text { recall }=\frac{(\mathrm{TP})}{(\mathrm{TP}+\mathrm{FN})} \\
& \text { precision }=\frac{(\mathrm{TP})}{(\mathrm{TP}+\mathrm{FP})} \\
& \mathrm{f}-\text { measure }=\frac{2 * \text { Recall } * \text { Precision }}{(\text { Recall }+ \text { Precision })}
\end{aligned}
$$

\section{Results and Discussions}

The Matlab $2015^{\mathrm{R}}$ programming platform that runs on the Windows 10 64bit operating system was used as the experimental base for this research. Endoscopic images used in this study have a total of 200 images with two categories: 100 endoscopic images of cancer category and 100 endoscopic images of the normal category. The dataset is divided into three parts to avoid bias on the results of artificial neural network training. The first part is the training dataset (70\%), the second part is the testing dataset $(15 \%)$, and the third part is the validation dataset (15\%). The default function Matlab 2015R (dividerand) is used as a dataset divider.

Feature extraction produces two kinds of features: morphological and spatial features. Morphological features produce information on circularity values. On the other hand, spatial features product information on energy values, contrast, correlation, and homogeneity. The values of spatial and morphological features are used as input from the artificial neural network classifier. Some morphological and spatial extraction values are presented in Table 2.

Although there are many algorithm choices available in the artificial neural network training process [19][20], this research uses Quasi-Newtonian (matlab: trainlm) algorithm because the QuasiNewtonian algorithm can produce an optimal artificial neural network learning process and faster to achieve generalization of output values compared to training algorithms such as Scaled-Conjugate or Resilient-Propagation [21]. The parameters of the artificial neural network (ANN) training process are presented in Table 3.

Training and testing are carried out in the Matlab R2016a environment that runs on an operating system platform on Windows 10 (64-bit) with an Intel® Core i5 ${ }^{\circledR} 4310$ processor computer; 8 GB Memory; Intel HD VGA Card. For simplification purpose, there will be presented only the results of 
Table 2. Spatial and morphological feature extraction result

\begin{tabular}{llllll}
\hline Image Condition & Energy & Contrast & Correlation & Homogeneity & Circularity \\
\hline Normal Image-1 & 0.18948 & 0.96020 & 0.20885 & 0.93779 & 0.95982 \\
Normal Image-2 & 0.18523 & 0.96077 & 0.17188 & 0.93618 & 0.93752 \\
Normal Image-3 & 0.19485 & 0.97017 & 0.21369 & 0.94112 & 0.85313 \\
Normal Image-4 & 0.20669 & 0.95677 & 0.17129 & 0.93349 & 0.77344 \\
Normal Image-5 & 0.16762 & 0.96966 & 0.17314 & 0.94374 & 0.90534 \\
Polyp Image-1 & 0.29893 & 0.94862 & 0.15432 & 0.91132 & 0.73008 \\
Polyp Image-2 & 0.20979 & 0.96249 & 0.21411 & 0.94192 & 0.91683 \\
Polyp Image-3 & 0.26171 & 0.96698 & 0.15399 & 0.92710 & 0.79852 \\
Polyp Image-4 & 0.24069 & 0.95111 & 0.18719 & 0.92481 & 0.66581 \\
Polyp Image-5 & 0.29893 & 0.94862 & 0.15432 & 0.91132 & 0.82152 \\
\hline
\end{tabular}

Table 3. ANN training set parameters

\begin{tabular}{lll}
\hline No & Parameter & Value \\
\hline 1. & Epoch & 25,000 \\
2. & Performance Function & mse (mean squared error) \\
3. & Goal & 0.01 \\
4. & Max. Fail & 6 (Matlab default) \\
5. & Min. Gradient & $1.00 \mathrm{e}^{-07}$ \\
6. & $\mu$ & $1.00 \mathrm{e}^{10}$ \\
\hline
\end{tabular}

the artificial neural network training process with 5-(11)-2 architecture with blended input feature (spatial and morphological) are presented in Fig. 5

From Fig. 6 it can be seen that the training process does not experience overfitting conditions. The absence of overfitting is indicated by the blue line $=$ train; green $=$ validation; red $=$ test that decreases simultaneously and does not intersect each other. A summary of the metrics for the results of artificial neural network training is presented in Table 4. A summary of the confusion matrix of the artificial neural network classifier for blended (spatial and morphological) input features is presented in Table 5. The summary of the confusion matrix of the artificial neural network classifier for the non-blended (spatial only) input feature is presented in Table 6.

The artificial neural network training process with a blended (spatial and morphological) input feature produces a regression value of 0.97232 presented in Fig. 6. Otherwise, the artificial neural network training process with a non-blended (spatial only) input feature produces 0.89151 regression value.

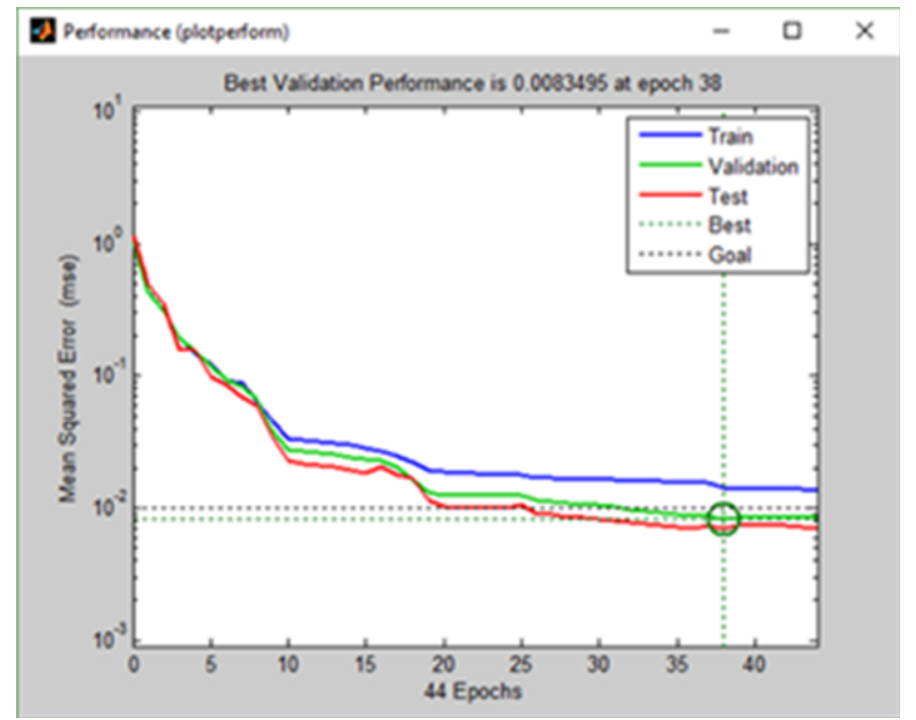

Fig. 5. Artificial neural network training result for blended feature 
Table 4. Summary of artificial neural network training

\begin{tabular}{llll}
\hline No & Parameter & Original Set & After Training \\
\hline 1. & Epoch & 25,000 & 14,218 \\
2. & Performance Function & mse (mean squared error) & Mse \\
3. & Goal & 0.01 & 0.00969 \\
4. & Max. Fail & 6 (Matlab default) & 2 \\
5. & Min. Gradient & $1.00 \mathrm{e}-07$ & $1.00 \mathrm{e}^{-07}$ \\
6. & $\mu$ & $1.00 \mathrm{e} 10$ & $1.00 \mathrm{e}^{10}$ \\
\hline
\end{tabular}

Table 5. Confusion matrix for blended (spatial and morphological) input feature

\begin{tabular}{llll}
\hline \multirow{2}{*}{ Image Condition } & \multicolumn{1}{c}{ Detected as } & Total \\
\cline { 2 - 3 } & Cancer & Normal & 100 \\
\hline Cancer & $94(\mathrm{TP})$ & $6(\mathrm{FP})$ & 100 \\
Normal & $8(\mathrm{FN})$ & $92(\mathrm{TN})$ & $\sum=200$ \\
\hline Total & & & $\sum$ \\
\hline
\end{tabular}

Table 6. Confusion matrix for non-blended (spatial only) input feature

\begin{tabular}{llll}
\hline \multirow{2}{*}{ Image Condition } & \multicolumn{2}{l}{ Detected as } & Total \\
\cline { 2 - 4 } & Cancer & Normal & 100 \\
Cancer & $89(\mathrm{TP})$ & $11(\mathrm{FP})$ & 100 \\
\hline Normal & $16(\mathrm{FN})$ & $84(\mathrm{TN})$ & $\sum=200$ \\
\hline Total & & & $\sum$ \\
\hline
\end{tabular}

Based on Table 5 and Table 6, it can be evaluated the performance of artificial neural network classifiers with blended and non-blended input features with indicators of accuracy, recall, precision, and f-measure. The values of the performance indicators artificial neural network classifiers with blended input feature, derived from Table 5 and Table 6 are presented in Table 7. For ease of use, a comparison from Table 7 also presented on Fig. 7.

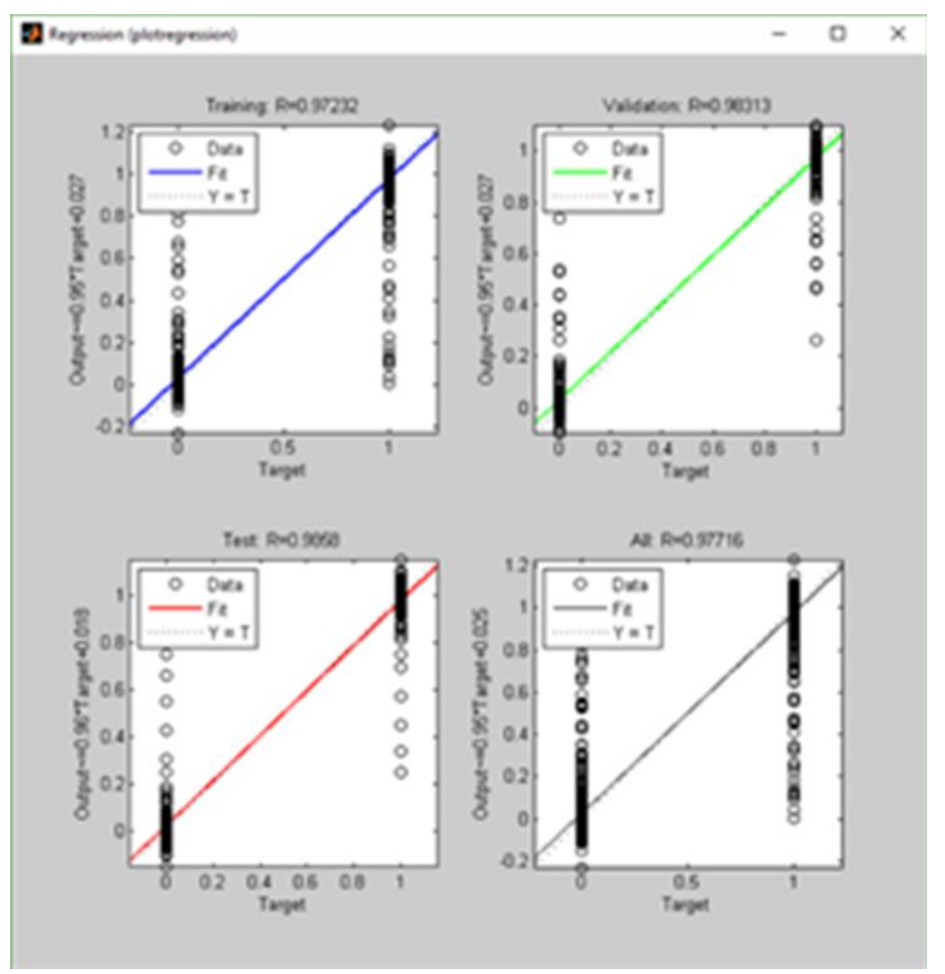

Fig. 6. Artificial neural network training regression result (blended input feature) 
Table 7. Artificial neural network classifier performace indicator

\begin{tabular}{lllll}
\hline \multirow{2}{*}{ No } & \multirow{2}{*}{ Perf. Indicator } & Input Feature & Gap \\
\cline { 3 - 5 } & & Blended & Non-blended & \\
\hline 1. & Accuracy & $0.930(93.0 \%)$ & $0.865(86.5 \%)$ & $0.065(6.5 \%)$ \\
2. & Recall & $0.921(92.1 \%)$ & $0.847(84.7 \%)$ & $0.074(7.4 \%)$ \\
3. & Precision & $0.940(94.0 \%)$ & $0.890(89.0 \%)$ & $0.050(5.0 \%)$ \\
4. & f-Measure & $0.930(93.0 \%)$ & $0.867(86.7 \%)$ & $0.063(6.3 \%)$ \\
\hline
\end{tabular}

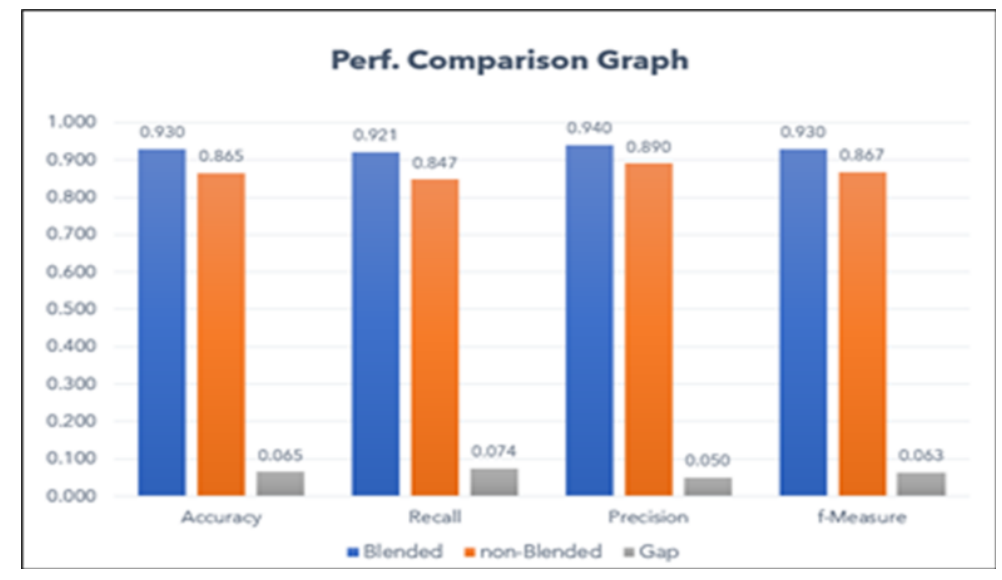

Fig. 7. Comparison graph

\section{Conclusion}

Identification of human intestinal conditions using an artificial neural network method with the blended input feature produces a higher accuracy value compared to the artificial neural network with the non-blended input feature. The difference in classifier performance produced between the two is quite significant, that is equal to 0.065 (6.5\%) for accuracy; $0.074(7.4 \%)$ for recall; 0.05 $(5.0 \%)$ for precision; and $0.063(6.3 \%)$ for f-measure. So it can be concluded that the use of blended features as neural network inputs sufficiently influences the results of identification of the condition of the human intestine.

\section{Acknowledgment}

Many thanks to Dr. Putut Bayupurnama Sp.PD from Internal Medicine Laboratory of Dr. Sardjito Hospital, Yogyakarta-Indonesia, were pleased to provide colorectal endoscopic image data, which is done as the basis of this research.

\section{Declarations}

\section{Author contribution}

All authors contributed equally as the main contributor of this paper. All authors read and approved the final paper.

\section{Funding statement}

This research did not receive any specific grant from funding agencies in the public, commercial, or not-for-profit sectors.

\section{Conflict of interest}

The authors declare no conflict of interest.

\section{Additional information}

No additional information is available for this paper. 


\section{References}

[1] N. Sengar, N. Mishra, M. K. Dutta, J. Prinosil, and R. Burget, "Grading of colorectal cancer using histology images," 2016 39th Int. Conf. Telecommun. Signal Process. TSP 2016, pp. 529-532, 2016, doi: 10.1109/TSP.2016.7760936.

[2] A. Ratheesh, P. Soman, M. Revathy Nair, R. G. Devika, and R. P. Aneesh, "Advanced algorithm for polyp detection using depth segmentation in colon endoscopy," 2016 Int. Conf. Commun. Syst. Networks, ComNet 2016, no. July, pp. 179-183, 2017, doi: 10.1109/CSN.2016.7824010.

[3] U. Athiyah, I. Muhimmah, and E. Marfianti, "Ekstraksi Ciri Polip dan Pendarahan Berdasarkan Citra Endoskopi Kolorektal," J. Inform. J. Pengemb. IT, vol. 3, no. 1, pp. 81-85, 2018.

[4] Y. Shin, H. A. Qadir, and I. Balasingham, "Abnormal colon polyp image synthesis using conditional adversarial networks for improved detection performance," IEEE Access, vol. 6, pp. 56007-56017, 2018, doi: 10.1109/ACCESS.2018.2872717.

[5] X. Wei, J. Xie, W. He, M. Min, Z. Ma, and J. Guo, "Quantitative Comparisons of Linked Color Imaging and WhiteLight Colonoscopy for Colorectal Polyp Analysis," Proc. 2018 6th IEEE Int. Conf. Netw. Infrastruct. Digit. Content, IC-NIDC 2018, pp. 140-144, 2018, doi: 10.1109/ICNIDC.2018.8525753.

[6] G. Tarik, A. Khalid, K. Jamal, and D. A. Benajah, "Polyps's region of interest detection in colonoscopy images by using clustering segmentation and region growing," Colloq. Inf. Sci. Technol. Cist, pp. 455-459, 2017, doi: 10.1109/CIST.2016.7805090.

[7] O. Bardhi, D. Sierra-Sosa, B. Garcia-Zapirain, and A. Elmaghraby, "Automatic colon polyp detection using Convolutional encoder-decoder model," 2017 IEEE Int. Symp. Signal Process. Inf. Technol. ISSPIT 2017, pp. 445448, 2018, doi: 10.1109/ISSPIT.2017.8388684.

[8] Q. Li et al., "Colorectal polyp segmentation using a fully convolutional neural network," Proc. - 2017 10th Int. Congr. Image Signal Process. Biomed. Eng. Informatics, CISP-BMEI 2017, vol. 2018-Janua, pp. 1-5, 2018, doi: 10.1109/CISP-BMEI.2017.8301980.

[9] I. O. Petre and C. Buiu, "A colon cancer microarray analysis technique," 2017 E-Health Bioeng. Conf. EHB 2017, pp. 265-268, 2017, doi: 10.1109/EHB.2017.7995412.

[10] N. Tajbakhsh, S. R. Gurudu, and J. Liang, “Automated polyp detection in colonoscopy videos using shape and context information," IEEE Trans. Med. Imaging, vol. 35, no. 2, pp. 630-644, 2016, doi: 10.1109/TMI.2015.2487997.

[11] Y. Hu et al., "Texture Feature Extraction and Analysis for Polyp Differentiation via Computed Tomography Colonography," IEEE Trans. Med. Imaging, vol. 35, no. 6, pp. 1522-1531, 2016, doi: 10.1109/TMI.2016.2518958.

[12] A. W. Muhammad, G. W. Sasmito, and I. Riadi, "Colorectal Polyp Detection Using Feedforward Neural Network with Image Feature Selection," Proceeding - 2018 Int. Symp. Adv. Intell. Informatics Revolutionize Intell. Informatics Spectr. Humanit. SAIN 2018, pp. 26-31, 2019, doi: 10.1109/SAIN.2018.8673371.

[13] S. Dutta, P. Sasmal, M. K. Bhuyan, and Y. Iwahori, "Automatic Segmentation of Polyps in Endoscopic Image Using Level-Set Formulation,” 2018 Int. Conf. Wirel. Commun. Signal Process. Networking, WiSPNET 2018, pp. 1-5, 2018, doi: 10.1109/WiSPNET.2018.8538615.

[14] J. Qu, N. Hiruta, K. Terai, H. Nosato, M. Murakawa, and H. Sakanashi, "Gastric Pathology Image Classification Using Stepwise Fine-Tuning for Deep Neural Networks," J. Healthc. Eng., vol. 2018, 2018, doi: 10.1155/2018/8961781.

[15] A. K. Palit and D. Popovic, Computational Intelligence in Time Series Forecasting, Advances in Industrial Control, 2005.

[16] Y. H. Hu and J.-N. Hwang, "Handbook of Neural Network Signal Processing." CRC Press, London, United Kingdom, 2002.

[17] L. C. Jain, "Recent Advances in Artificial Neural Networks," Recent Adv. Artif. Neural Networks, 2018, doi: $10.1201 / 9781351076210$.

[18] A. Geron, Hands-On Machine Learing With Scikit-Learn \& Tensor Flow. O’Reilly Media, 2017.

[19] I. Riadi, Sunardi, and A. W. Muhammad, "DDoS Detection Using Artificial Neural Network Regarding Variation of Training Function,” Adv. Sci. Lett., vol. 24, no. 12, pp. 9163-9167, 2018, doi: 10.1166/asl.2018.12117.

[20] I. Riadi, A. Wirawan, and S. -, "Network Packet Classification using Neural Network based on Training Function and Hidden Layer Neuron Number Variation," Int. J. Adv. Comput. Sci. Appl., vol. 8, no. 6, pp. 248-252, 2017, doi: 10.14569/ijacsa.2017.080631.

[21] A. Azhari, A. W. Muhammad, and C. F. M. Foozy, "Machine Learning-Based Distributed Denial of Service Attack Detection on Intrusion Detection System Regarding to Feature Selection," International Journal of Artificial Intelligence Research, vol. 4, no. 1, Feb. 2020. 